OPEN ACCESS

Edited by: James P. O'Gara, National University of Ireland Galway, Ireland

Reviewed by: lan Monk,

Trinity College, Dublin, Ireland Marta Zapotoczna,

Trinity College, Dublin, Ireland

${ }^{*}$ Correspondence: Eric W. Triplett ewt@ufl.edu

Specialty section:

This article was submitted to Infectious Diseases,

a section of the journal

Frontiers in Microbiology

Received: 30 August 2017 Accepted: 30 November 2017 Published: 14 December 2017

Citation:

Rodriguez MD, Paul Z, Wood CE, Rice KC and Triplett EW (2017) Construction of Stable Fluorescent Reporter Plasmids for Use in Staphylococcus aureus.

Front. Microbiol. 8:2491. doi: 10.3389/fmicb.2017.02491

\section{Construction of Stable Fluorescent Reporter Plasmids for Use in Staphylococcus aureus}

\author{
Michelle D. Rodriguez ${ }^{1}$, Zubin Paul'2, Charles E. Wood ${ }^{2}$, Kelly C. Rice ${ }^{1}$ and \\ Eric W. Triplett ${ }^{1 *}$
}

${ }^{1}$ Department of Microbiology and Cell Science, Institute of Food and Agricultural Sciences, University of Florida, Gainesville, FL, United States, ${ }^{2}$ Department of Physiology and Functional Genomics, College of Medicine, University of Florida, Gainesville, FL, United States

Here, the genes encoding three different fluorescent proteins were cloned into the stably maintained Staphylococcus aureus shuttle vector pKK30. The resulting plasmids were transformed into two S. aureus strains; SH1000 and RN4220. Stability assays illustrated that the three recombinant plasmids retained near 100\% maintenance in vitro for 160 generations. S. aureus strain SH1000 expressing green fluorescent protein was then inoculated in an ovine model and in vivo stability for 6 days was demonstrated. In essence, these reporter plasmids represent a useful set of tools for dynamic imaging studies in S. aureus. These three reporter plasmids are available through BEI Resources.

Keywords: : Staphylococcus aureus, fluorescence, plasmid, stable, inoculation

\section{INTRODUCTION}

Instability of recombinant plasmids has posed a major burden in studies on staphylococci, especially with regard to in vivo and immune cell interaction experiments where antibiotic treatment is difficult. The feeding of animals with antibiotics to maintain plasmid stability may not be sufficient to confer plasmid stability in all infection sites. The use of antibiotics may also lead to unwanted perturbations in the host microbiota, leading to confounding results in microbiome studies (Kamruzzaman et al., 2017). In addition, certain classes of antibiotics cannot effectively cross host membranes and are ineffective in eukaryotic systems. Designing plasmids that persist both in in vivo and in vitvo conditions remains a challenge in Staphylococcus aureus research (Krute et al., 2016). Fortunately, plasmids based on the LAC-p01 shuttle vector, pKK30 and pKK22, that are stable in the absence of selection pressure are available (Krute et al., 2016), thereby eliminating the need for antibiotic use during the course of in vivo and in vitro experiments. Here, the construction of fluorescent reporter plasmids are described using pKK30 as a backbone because it lacks the four predicted open reading frames for use in non-USA300 S. aureus isolates (Krute et al., 2016). Three fluorescent reporter gene inserts were ligated into pKK30 including superfolder $g f p$, dsRed, and FP650 (far red), the latter two of which were previously codon-optimized for S. aureus (Bose et al., 2013). The fluorescent protein genes were cloned downstream of the sarAP1 promoter - dfrA gene fusion and then electroporated into S. aureus RN4220 followed by S. aureus RN4220, plasmid extracted and electroportated into SH1000. 


\section{MATERIALS AND METHODS}

\section{Bacterial Strains and Media}

Plasmids pGFP-F, pRFP-F, and pFP650-F were obtained from BEI Resources [Nebraska Transposon Mutant Library (NTML) Genetic Toolbox, NR-49947]. S. aureus strains containing pRFP, pGFP, and pFP650 were cultured in tryptic soy agar supplemented with $5 \mathrm{ug} / \mathrm{mL}$ chloramphenicol for selection. For cloning purposes, Escherichia coli DH5a (for pCR-Blunt-based cloning) and DH5 $\alpha \lambda$ pir (for propogation of pKK30 and derivatives) were cultured in Luria-Bertani broth supplemented with $50 \mu \mathrm{g} / \mathrm{ml}$ kanamycin or $10 \mu \mathrm{g} / \mathrm{mL}$ trimethoprim, as appropriate. $S$. aureus cells were made competent for transformation as described previously (Bose, 2014). The bacterial strains used in this study are listed in Table 1.

\section{Plasmid Purification and PCR}

Plasmids were purified from $S$. aureus and E. coli cultures using a Qiagen Plasmid Mini Kit (Qiagen, Inc., Valencia, CA, United States). S. aureus cell suspensions were pretreated with $1 \mu \mathrm{L}$ of $2 \mathrm{mg} / \mathrm{mL}$ lysostaphin (Sigma-Aldrich) for $30 \mathrm{~min}$ at $37^{\circ} \mathrm{C}$. DNA was quantified using a Nanodrop spectrophotometer (Thermo Scientific, Wilmington, DE, United States), and fragment sizes were assessed on a $1 \%$ agarose gel. All PCR reactions were performed with an Applied Biosystems GeneAmp PCR System 9700 (Life Technologies, Corp., Carlsbald, CA, United States) for appropriate primer sets on each template (Bose et al., 2013) using proofreading polymerase Accuprime Pfx (Invitrogen). PCR reactions were prepared with $50 \mu \mathrm{L}$

TABLE 1 | Bacterial strains and plasmids used in this study.

Strain or plasmid
Bacterial strains
Escherichia coli DH5 $\alpha$
E. coli DH5 $\alpha \lambda$ pir
E. coli DH5 $\alpha$ ipir (pSGFPS1)
E. coli DH5 $\alpha \lambda$ pir (pSRFPS1)
E. coli DH5 $\alpha$ ipir (pSFRFPS1)
S. aureus RN4220
S. aureus SH1000
S. aureus RN4220
(pSGFPS1)
S. aureus RN4220
(pSRFPS1)
S. aureus RN4220
(pSFRFPS1)
S. aureus SH1000
(pSGFPS1)
S. aureus SH1000
(pSRFPS1)
S. aureus SH1000
(pSFRFPS1)

\section{Description}

Strain used for cloning purposes

Strain used for pKK30 cloning and maintenance

GFP-labeled DH5 $\alpha \lambda$ pir

RFP-labeled DH5 $\alpha \lambda$ pir

FRFP-labeled DH5 $\alpha \lambda$ pir

Highly mutagenized, transformable $S$. aureus

Wild-type $S$. aureus derived from 8325-4 lineage

GFP-labeled RN4220

RFP-labeled RN4220

FRFP-labeled RN4220

GFP-labeled SH1000

RFP-labeled SH1000

FRFP-labeled SH100O
Source

Silhavy

et al., 1984

Krute et al.,

2016

This study

This study

This study

Kreiswirth

et al., 1983

Horsburgh

et al., 2002

This study

This study

This study

This study

This study

This study 100x Accuprime mix, $1 \mu \mathrm{L}$ of $10 \mu \mathrm{M}$ primer stock, $20 \mathrm{ng}$ of plasmid DNA adjusted to $50 \mu \mathrm{L}$ total volume with sterile, nuclease-free water. Cycles consisted of a 2 min denaturation step at $95^{\circ} \mathrm{C}$ followed by 30 cycles of $15 \mathrm{~s}$ at $95^{\circ} \mathrm{C}$ for further denaturation, $30 \mathrm{~s}$ at $60^{\circ} \mathrm{C}$ for annealing, $1 \mathrm{~min}$ at $72^{\circ} \mathrm{C}$ extension and ended with a $10 \mathrm{~min}$ extension at $72^{\circ} \mathrm{C}$. PCR products were purified using PCR Purification kit (Qiagen, Hilden, Germany), quantified fluorometrically by QuBit dsDNA High Sensitivity (Invitrogen, Life Technologies, Inc., Carlsbad, CA, United States), and fragments size was assessed on an $1 \%$ agarose gel. The DsRed and eqFP650 reporter genes used were codon-optimized for $S$. aureus and synthesized by Invitrogen. Synthesized genes were amplified by PCR from pTnT plasmids using the primer sets JBTN18/JBTN19, JBTN20/JBTN21 for DsRed, and eqFP650 as previously described (Bose et al., 2013). The gene encoding superfolder green fluorescent protein was amplified from pJB68 using the primer pair JBTN37/JBTN38, respectively. All fluorescent gene primers included a nonhomologous AscI sequence (GGCGCGCC), to allow cloning of fluorescent genes downstream of the $d f r A$ cassette (Table 2).

\section{Construction of Reporter Plasmids into S. aureus RN4220 and SH1000}

The pKK30 plasmid described previously (Krute et al., 2016) was used as the backbone for these new plasmids. In addition, pKK30 includes the selectable marker, $d f r A$, which is under the control of the constitutive promoter (sarAP1). This marker confers trimethoprim resistance in these $S$. aureus strains. Furthermore, the blaZ transcriptional terminator in pKK30 prevents transcription beyond the reporter gene inserts, allowing for enhanced plasmid stability and maintenance.

Blunt ends of GFP, DsRED, and FP650 were generated for amplified fluorescent reporter genes and ligated into pCRBlunt vector using the Zero Blunt TOPO PCR Cloning Kit (Invitrogen). Approximately, $5 \mu \mathrm{L}$ of each ligation reaction was transformed into E. coli DH5a by heat shock and plated on LB plates supplemented with $50 \mu \mathrm{g} / \mathrm{mL}$ kanamycin for selection.

TABLE 2 | Oligonucleotides used in this study.

\begin{tabular}{llc}
\hline Oligonucleotide & Sequence $\left(\mathbf{5}^{\prime}-\mathbf{3}^{\prime}\right)^{*}$ & Source \\
\hline JBTN18 & ggcgcgccTGATTAACTTATAAGGAGG & This study \\
& AAAAACATATGGA &
\end{tabular}

JBTN19 ggcgcgccTTATAAAAACAAATGATGAC This study

JBTN20 ggcgegccTGATTAACTITATAAGGAGG This study

AAAAACATATGGG

JBTN21 ggcgcgcCTTAACTATGACCTAATIT $\quad$ This study

JBTN37 ggcgcgccTGATTAACTTATAAGGAGG This study

JBTN38 ggcgcgcCTTATTGTAGAGCTCAT This study CCATGCCATGTG

dfrA-pKK30 TTGTCGCTCACGATAAACAAA This study

* Lowercase lettering denotes Ascl site to promote cloning into Ascl insertion site on $\mathrm{pKK} 30$. 
TABLE 3 | In vitro stability of pkk30 reporter plasmids.

\begin{tabular}{|c|c|c|c|c|c|}
\hline Plasmid & Strain & Trial & Generations & Plasmid present* & $\%$ with plasmid \\
\hline \multirow[t]{3}{*}{ pSRFPS } & S. aureus SH1000 & 1 & 160 & $145 / 150$ & 97 \\
\hline & & 2 & 160 & $140 / 150$ & 93 \\
\hline & & 3 & 160 & $150 / 150$ & 100 \\
\hline \multirow[t]{3}{*}{ pSGFPS } & S. aureus $\mathrm{SH} 1000$ & 1 & 160 & $150 / 150$ & 100 \\
\hline & & 2 & 160 & $150 / 150$ & 100 \\
\hline & & 3 & 160 & $150 / 150$ & 100 \\
\hline \multirow[t]{3}{*}{ pSFRFPS } & S. aureus SH1000 & 1 & 160 & $150 / 150$ & 100 \\
\hline & & 2 & 160 & $150 / 150$ & 100 \\
\hline & & 3 & 160 & $148 / 150$ & 99 \\
\hline \multirow[t]{3}{*}{ pSRFPS } & S. aureus RN4220 & 1 & 160 & $150 / 150$ & 100 \\
\hline & & 2 & 160 & $150 / 150$ & 100 \\
\hline & & 3 & 160 & $150 / 150$ & 100 \\
\hline \multirow[t]{3}{*}{ pSGFPS } & S. aureus RN4220 & 1 & 160 & $150 / 150$ & 100 \\
\hline & & 2 & 160 & $149 / 150$ & 99 \\
\hline & & 3 & 160 & $146 / 150$ & 97 \\
\hline \multirow[t]{3}{*}{ pSFRFPS } & S. aureus RN4220 & 1 & 160 & $150 / 150$ & 100 \\
\hline & & 2 & 160 & $148 / 150$ & 99 \\
\hline & & 3 & 160 & $150 / 150$ & 100 \\
\hline
\end{tabular}

*Screened 150 colonies per plated culture.

Resulting colonies were then screened for the presence of each fluorescent gene insert by PCR using the primers described above. Subsequently, pCR-Blunt containing fluorescent gene DNA was digested with AscI, the appropriately sized restriction fragments were gel purified, ligated, and dephosphorylated, AscIdigested pKK30. By amplification with reporter gene using the primers described above as well as the $d f r A$ primer from $\mathrm{pKK} 30$, $5^{\prime}$-TTGTCGCTCACGATAAACAAA-3'. The ligation products were transformed into $E$. coli $\mathrm{DH} 5 \alpha \lambda$ pir and then screened for the correct presence and orientation of reporter gene inserts by PCR using a forward primer specific for the $d f r A$ gene (5'-TTGTCGCTCACGATAAACAAA-3') and the appropriate reverse primer for each reporter gene. Proper orientation of inserts was also confirmed by Sanger sequencing downstream from the $d f r A$ insertion site. The $E$. coli strains containing each plasmid were then stored in $-80^{\circ} \mathrm{C}$ in $50 \%$ glycerol. The three fluorescent inserts were used to construct pSGFPS1, pSRFPS1, and pSFRFPS1. The nucleotide sequences of pSFRFPS, pSGFPS1, pSRFPS1, and can be obtained from GenBank accession numbers MF769789, MF769790, and MF769791, respectively. Collectively, these plasmids are referred to as pSxFPS1.

\section{Electroporation of Reporter Plasmids into S. aureus SH1000 and RN4220}

Electrocompetent cells were prepared as described previously (Bose, 2014). Approximately, $1 \mu \mathrm{g}$ of pSxFPS1 DNA derived from E. coli $\mathrm{DH} 5 \mathrm{a} \lambda$ pir was mixed with $S$. aureus electrocompetent cells and pulsed at $2.3 \mathrm{kV}, 100 \Omega$, and $25 \mu \mathrm{F}$ using the Gene
Pulser Xcell Electroporation System (Bio-Rad Laboratories). Next, B2 broth (Bose, 2014) was added and cuvettes were incubated for $2 \mathrm{~h}$ at $37^{\circ} \mathrm{C}$. Cell suspensions were then spread on solid TSA with $10 \mu \mathrm{g} / \mathrm{mL}$ trimethoprim, and incubated overnight at $37^{\circ} \mathrm{C}$. Transformation efficiencies were determined for all three pSxFPS1 plasmids. For the RN4220 strains, the transformation efficiencies were $1.075 \times 10^{3}, 5.34 \times 10^{5}$, and $4.88 \times 10^{5}$ per $\mathrm{ng} / \mathrm{DNA}$ for pSGFPS1, pSRFPS1, pSFRFPS, respectively. For the SH1000 strains, plasmids were first extracted from RN4220 and these were used to transform SH1000 with the transformation efficiencies of $6.45 \times 10^{2}$, $5.37 \times 10^{5}$, and $3.9 \times 10^{5}$ per $n g / D N A$ for pSGFPS1, pSRFPS1, pSFRFPS, respectively. Proper orientation of the inserts was firmed by PCR and sequencing. The presence of the reporter genes was confirmed by fluorescence microscopy. Transformants were confirmed by assessing proper orientation with isolate sequencing as described above and the presence of reporter gene inserts were observed visually by fluorescence microscopy (EVOS FL Cell Imaging System, ThermoFisher). RFP was excited at $531 \mathrm{~nm}$ and emission detected at $593 \mathrm{~nm}$. GFP was excited at $470 \mathrm{~nm}$ and emission detected at $510 \mathrm{~nm}$. FP650 was excited at $592 \mathrm{~nm}$ and emission detected at $650 \mathrm{~nm}$.

\section{In Vitro Stability Assays on pSxFPS1 Reporter Plasmids}

Long-term stability assays were performed, as described by Bose (2014), on the three pSxFPS1 reporter plasmids, and pKK30 as 


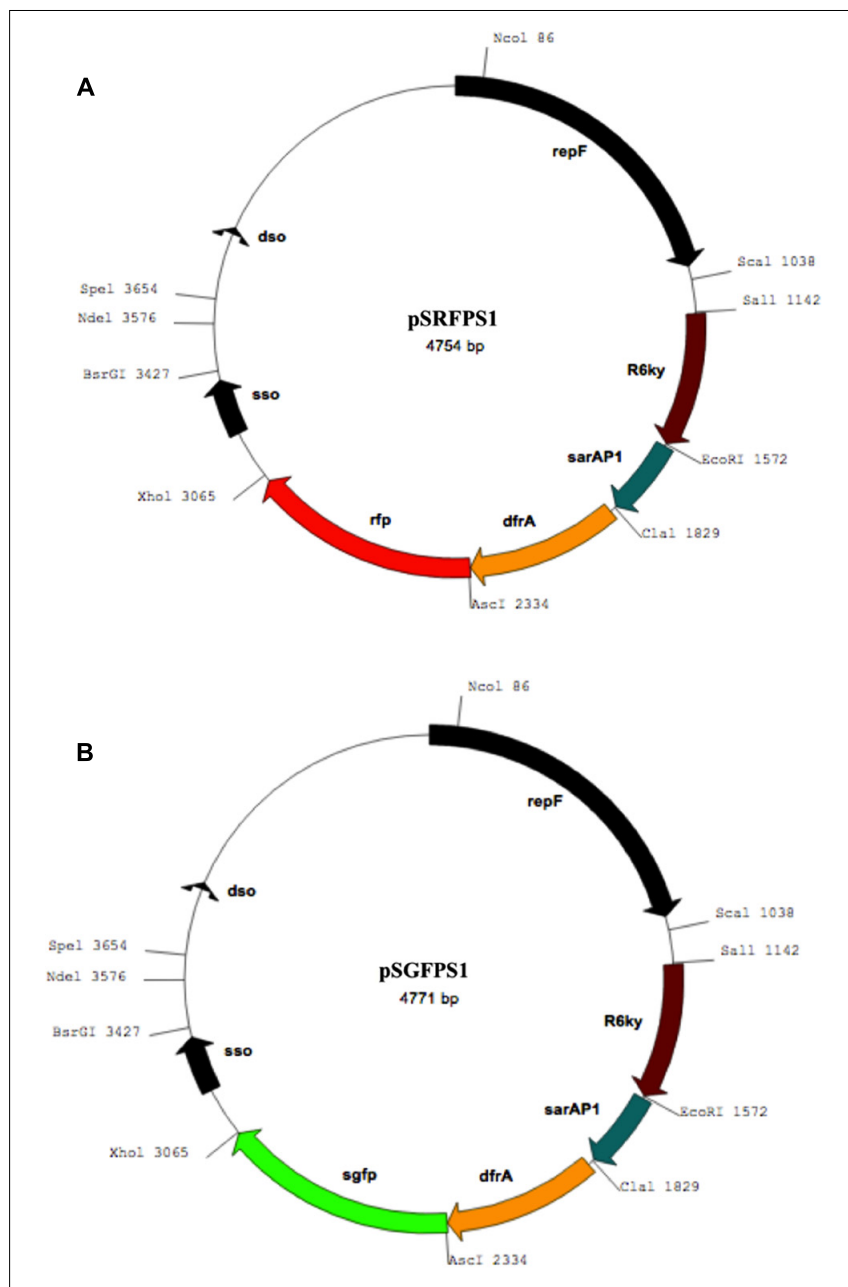

C

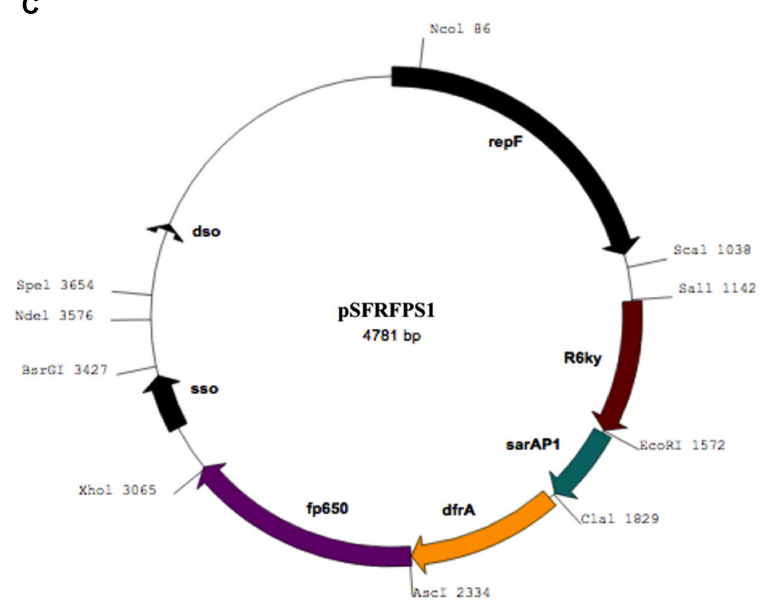

FIGURE 1 | Maps of recombinant plasmids containing genes coding for (A) red (pSRFPS1), (B) green (pSGFPS1), and (C) far-red (pSFRFPS1) fluorescent proteins.

a control, to determine maintenance of plasmid during noncontinuous exponential phase in the absence of trimethoprim in S. aureus strains RN4220 and SH1000. Recombinant SH1000 and RN4220 strains were inoculated in $10 \mathrm{~mL}$ TSB supplemented with $10 \mu \mathrm{g} / \mathrm{ml}$ trimethoprim, and grown overnight at $37^{\circ} \mathrm{C}$ with shaking $(250 \mathrm{rpm})$. Next, cultures were diluted to $10^{-4}$ in $10 \mathrm{~mL}$ TSB without trimethoprim in $125 \mathrm{~mL}$ flasks and cultured at $37^{\circ} \mathrm{C}$ at $250 \mathrm{rpm}$ up to an $\mathrm{OD}_{600}$ of 1.5 . Cultures were then serially diluted to $10^{-7}$ in $10 \mathrm{~mL}$ TSB, $100 \mu \mathrm{L}$ aliquots were spread plated onto TSA without antibiotic, and incubated overnight. Resulting colonies were then patched on to antibiotic plates to determine the percentage of colonies carrying pSxFPS1 or pKK30 (Table 3). These steps were continued for four additional days with the last step being plating and patching on to media with trimethoprim. The number of generations was calculated using $\mathrm{OD}_{600}$ readings and dilution factor, resulting in approximately 160 generations observed for all eight recombinant strains including those with pKK30. In a parallel experiment to assess the effect of each plasmid on growth of the host bacterium, overnight cultures of RN4220 and SH1000 (containing one of the reporter plasmids or pKK30) were diluted to an $\mathrm{OD}_{600}$ of 0.1 in $200 \mu \mathrm{L}$ of tryptic soy broth in triplicate on 96-well plates alongside positive and negative controls. For continued bacterial culture growth, plates were incubated in a spectrophotometer at $250 \mathrm{rpm}$ for $12 \mathrm{~h}$ to assess exponential phase growth in the absence of trimethoprim. This experiment was repeated three times and the mean of the three data points for each growth point were used for the analysis (Figure 2).

\section{Inoculation of an Ovine Model with Fluorescent S. aureus SH1000}

An ovine model was used for this work because our long-term objective is to understand the role of bacteria, if any, in premature birth. Because of its size, sheep are considered an excellent model for premature birth. All procedures were approved by the University of Florida Animal Care and Use Committee and performed in accordance with the Guiding Principles for Use of Animals of the American Physiological Society. This work was approved by the University of Florida's Institutional Animal Care and Use Committee (IACUC) as part of study number 201508915. Pregnant ewes of known gestational age and mixed breeds were transported to the University of Florida and acclimatized to the housing, light-dark cycle, temperature, and diet of the laboratory environment. Ewes were fed a diet of pelleted feed according to NRC standards for the ewe's body weight and gestation. On days 130-132 of gestation, all ewes were inoculated intravenously with $S$. aureus SH1000 (pSGFPS1). The intravenous dose was $10^{4} \mathrm{cfu} / \mathrm{mL}(n=4)$. Four to six days after inoculation, the ewes were humanely sacrificed and tissues were recovered from mother, placenta, and fetus for analysis of GFP and for isolation and culture of $S$. aureus SH1000. Antibodies against GFP with DAB were used to visualize bacteria expressing fluorescent proteins in fetal and maternal tissues.

\section{Immunohistochemistry}

Tissue samples were fixed using $4 \%$ paraformaldehyde overnight after necropsy and set in $70 \%$ alcohol for storage. Tissue slices were cut to $2-3 \mathrm{~mm}$ slices and dehydrated in $90-100 \%$ Reagent 


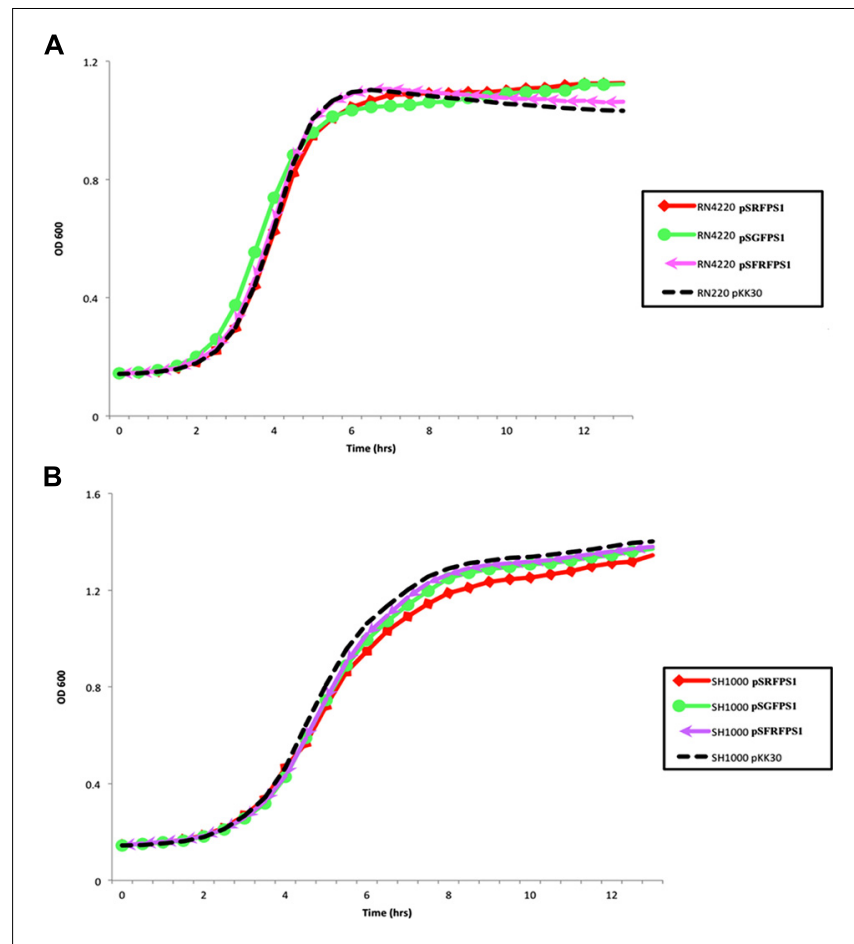

FIGURE 2 | (A) Growth of fluorescent strains containing pSGFPS1, pSRFPS1, and pSFRFPS1 in (A) RN4220 and (B) SH1000 in TSB media without antibiotic. Growth of pKK30 vector without inserts is shown in black.
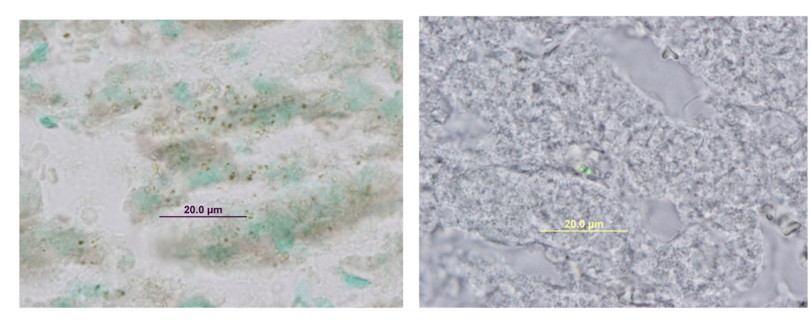

FIGURE 3 | Immunohistochemical staining with anti-GFP in maternal liver infected with Staphylococcus aureus SH1000 (pSGFPS1).

Alcohol overnight. Slices were then cleared in xylene for 23 h. Following xylene clearance, tissues were placed in liquid paraffin and set in the vacuum oven at $60^{\circ} \mathrm{C}$ for $1 \mathrm{~h}$ with the vacuum on and $1 \mathrm{~h}$ with the vacuum off. Tissue slices were then mounted in paraffin blocks and left to dry overnight. Sections were cut at 5-7 micron thickness and mounted on slides.

Tissue sections were hydrated in a graduated alcohol dilution, quenched with BLOXALL Blocking Solution for $10 \mathrm{~min}$, and blocked in 2.5\% horse serum (R.T.U. Vectastain Kit, PK7200) for $30 \mathrm{~min}$. Then, slides were incubated for $1 \mathrm{~h}$ with a $1 \mathrm{mg} / \mathrm{mL}$ anti-GFP antibody (Millipore AB3080) at a 1:500 dilution. Tissues were incubated for $30 \mathrm{~min}$ with a universal biotinylated antibody from the RTU Vectastain Kit. DAB (HRP) substrate (Vector SK-4100) was used for visualization of the anti-GFP antibody, and slides were counter stained with methyl green. Images were taken at 10 and 40x zoom using Olympus Digital Fluorescence microscope (Olympus Life Science).

\section{Culturing of Fluorescent S. aureus SH1000 from Ovine Tissues}

Following a 4-6 day incubation period, $100 \mathrm{mg}$ of maternal liver, lung, cerebral cortex, and placenta samples were mechanically homogenized in $500 \mu \mathrm{L}$ of Brain Heart Infusion Broth (SigmaAldrich) for $30 \mathrm{~s}$. Homogenates were serially diluted 10-fold, $100 \mu \mathrm{L}$ aliquots were spread-plated onto solid BHI medium, and incubated overnight at $37^{\circ} \mathrm{C}$. To assess abundance of SH1000 containing the three reporter plasmids, colonies on $10^{4} \mathrm{cfu} / \mathrm{mL}$ plates were enumerated, with resulting values of $1.9 \times 10^{7}$ $\mathrm{cfu} / \mathrm{mL}$ for pSGFPS1, $1.1 \times 10^{7} \mathrm{cfu} / \mathrm{mL}$ for pSRFPS1, and $1.3 \times 10^{7} \mathrm{cfu} / \mathrm{mL}$ for $\mathrm{pSFRFPS} 1$. Colonies were also screened by fluorescence microscopy as described previously, and plasmid DNA was sequenced to determine proper orientation of the sGFP insert.

\section{RESULTS}

\section{Stability of pSxFPS1 Reporter Plasmids in Vitro}

The resulting recombinant reporter plasmids (Figure 1) exhibited high stability in both $S$. aureus strains tested. The use of codon-optimized DsRed and eqFP650 proteins ensure desired brightness and detectability for fluorescent imaging techniques and histology work. Non-continuous exponential stability assays demonstrated that the pSxFPS1 reporter constructs remained stable in S. aureus SH1000 and RN4220 (Table 2). In both strains, the number of colonies on TSA without trimethoprim compared to the number on TSA with trimethoprim remained constant across all three trials. The presence of the plasmid in colonies cultured without antibiotic was confirmed by both fluorescence microscopy and PCR amplification sequencing. All three recombinant strains behaved similarly to pKK30 and were comparably stable, with an average of $99.1 \%$ of colonies cultured without trimethoprim capable of growth with the antibiotic across three replicate experients for each recombinant strain (Table 2).

\section{S. aureus Reporter Strains Were Cultured from Ovine Tissues}

Staphylococcus aureus SH1000 (pSGFPS1) was successfully recovered on solid media from maternal tissues following a 6 day incubation period in vivo. Bacterial colonies were highly abundant in the placenta, liver, and spleen samples. These cultures also exhibited equivalent brightness when assessed by fluorescent microscopy compared to the inoculum strains (Figure 2). Thus, viable, fluorescently labeled $S$. aureus can be cultured from infected animals. In addition to culturing, S. aureus (pSGFPS1) cells were detected in placenta and maternal liver (Figure 3). GFP-expressing bacteria were detectable in 
both of these tissues using immunohistochemistry using GFP antibodies (Figures 4, 5) and by fluorescence microscopy as described previously. At $10^{4}$ cells of S. aureus SH1000 with a fluorescent plasmid, no sickness or lethality were observed in the ewe or the fetus. However, in subsequent experiments, inoculation with $10^{6}$ cells caused illness in the ewe while a dose $10^{8}$ cells was lethal to the ewe and fetus. This result shows that SH1000 maintains virulence with the reporter plasmids in vivo.

\section{DISCUSSION}

The three stable reporter plasmids described here allow for the study of the movement and growth of $S$. aureus within animal hosts without the need for any disruption of the bacterial chromosome or the use of any antibiotics to maintain their presence in the bacterial cell. Each reporter plasmids contains a gene coding for a different fluorescent protein. Having these different labels, for example, allows an investigator to inoculate multiple sites simultaneously in order to test routes of infection and reduce the number of animals used in the experiment. The use of $S$. aureus in this study is preferrable due to its efficient colonization and virulence in vivo.

Plasmid stability in the absence of selection pressure is another crucial characteristic when the genes of interest are carried on an extrachromosomal replicon. This trait is conferred on these plasmids from the original pKK30 background and the stability phenotype is confirmed here in these new constructs. As a result of plasmid stability during cell division in the absence of selection pressure, an investigator can use a strain carrying any of these plasmids and be confident that the fluorescent cells observed in a tissue accurately reflects the population size in the tissue. Adding antibiotics to maintain plasmid stability can add a tremendous confounder to experiments where an investigator is interested in how a labeled bacterium may interact with the native bacterial populations. Fortunately, these plasmids remove that confounder.

In this study, labeled cells were visualized within sheep tissues 4-6 days after inoculation of the animal by immunocytochemistry and fluorescence microscopy. In addition, culturable $S$. aureus strains containing these plasmids were re-isolated from the sheep tissues 6 days after inoculation. A recent study showed that hypoxic treatment of pregnant ewes resulted in strains of $S$. aureus and $S$. simulans entering the fetal brain presumably through the placenta (Zarate et al., 2017). Although the genomes of the $S$. simulans strains isolated from the fetal brain and placenta were identical (within the limits of genome sequencing error), inoculation of the pregnant ewe with a Staphylococcus strain labeled with one of the plasmids described here would resolve the question of the origin of the strain in the fetal brain.
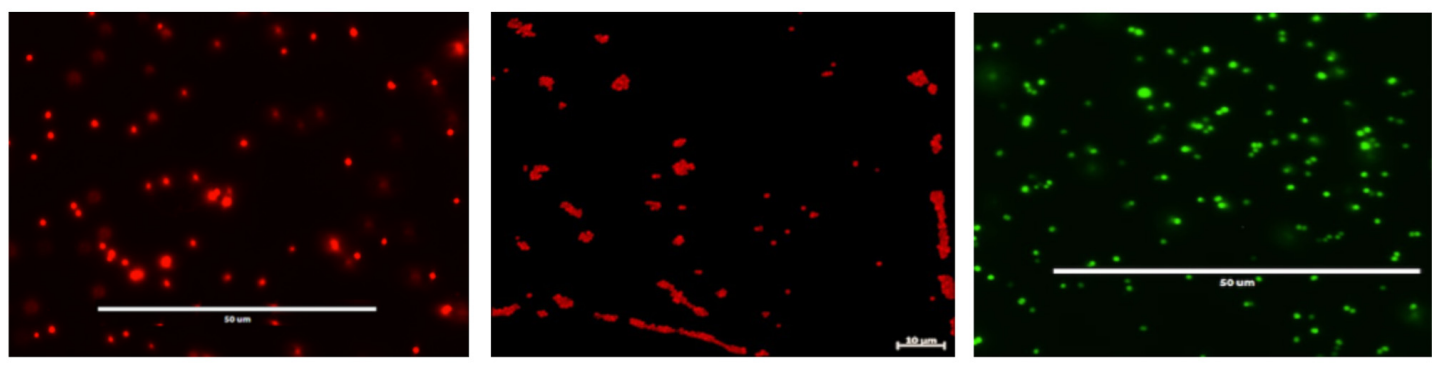

FIGURE 4 | Fluorescent microscopy of cultured S. aureus SH1000 strains containing pSRFPS1 (left), pSFRFPS1 (middle), and pSGFPS1 (right).
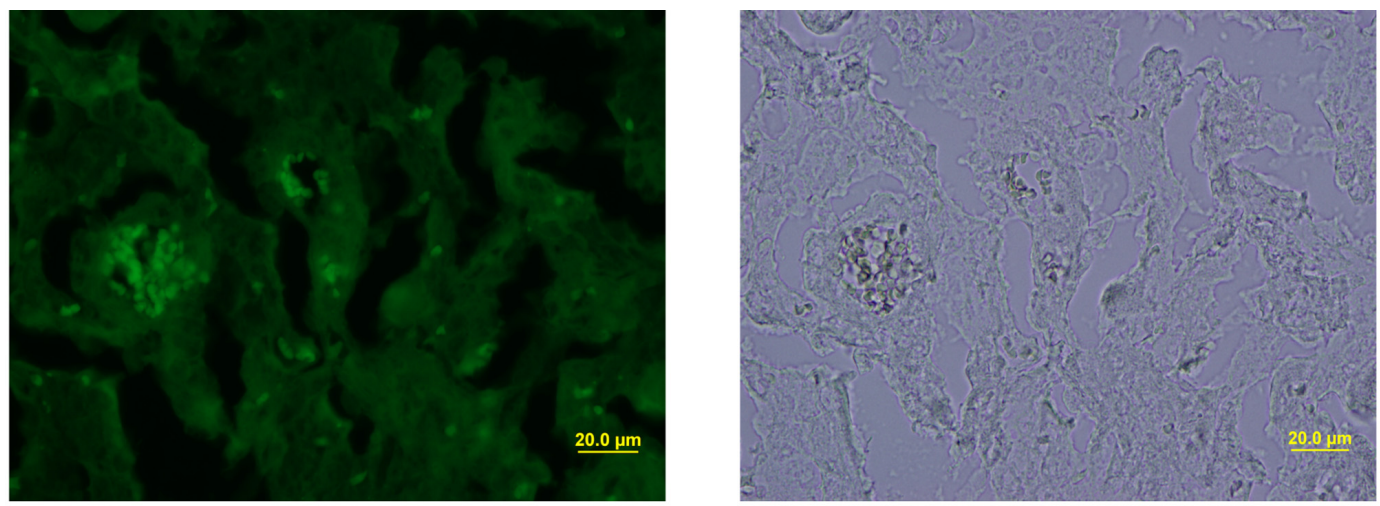

FIGURE 5 | Fluorescence microscopy and bright-field staining of maternal liver infected with S. aureus SH1000 (pSGFPS1). 
The National Institute of Allergy and Infectious Diseases of the National Institutes of Health are sufficiently persuaded by the utility of these $S$. aureus - optimized plasmids to make them available through BEI Resources. This will make these plasmids available to the entire community of microbiologists for the foreseeable future.

\section{AUTHOR CONTRIBUTIONS}

MR did the lab work needed for the project and wrote the first draft of the paper. ZP performed and interpreted the tissue staining done in this work. CW did the animal validation of the bacterial strains used here and edited the paper. KR provided valuable advice on the choice replicons and reporter genes used in this work. ET conceptualized the work, worked on all drafts of

\section{REFERENCES}

Bose, J. L. (2014). "Genetic manipulation of staphylococci," in Staphylococcus epidermidis: Methods and Protocols, Vol. 1106, ed. P. D. Fey (New York, NY: Humana Press), 101-111. doi: 10.1007/978-1-62703736-5_8

Bose, J. L., Fey, P. D., and Bayles, K. W. (2013). Genetic tools to enhance the study of gene function and regulation in Staphylococcus aureus. Appl. Environ. Microbiol. 79, 2218-2224. doi: 10.1128/aem.00136-13

Horsburgh, M. J., Aish, J. L., White, I. J., Shaw, L., Lithgow, J. K., and Foster, S. J. (2002). SigmaB modulates virulence determinant expression and stress resistance: characterization of a functional $r s b U$ strain derived from Staphylococcus aureus. J. Bacteriol. 184, 5457-5467. doi: 10.1128/JB.184.19. 5457-5467.2002

Kamruzzaman, M., Shoma, S., Thomas, C. M., Partridge, S. R., and Iredell, J. R. (2017). Plasmid interference for curing antibiotic resistance plasmids in vivo. PLOS ONE 12:e0172913. doi: 10.1371/journal.pone.01 72913

Kreiswirth, B. N., Lofdahl, S., Betley, M. J., O’Reilly, M., Schlievert, P. M., Bergdoll, M. S., et al. (1983). The toxic shock syndrome exotoxin structural gene is not detectably transmitted by a prophage. Nature 305, 709-712. doi: 10.1038/ $305709 \mathrm{a} 0$ the paper, obtained the accession numbers for the plasmids, and worked with BEI Resources to make the plasmids available to the research community.

\section{FUNDING}

This work was supported by the NIH NIAID, grant number 5R21AI120195-02 (CW and ET).

\section{ACKNOWLEDGMENT}

The authors thank Dr. Jeffrey Bose (Kansas University Medical Center) for providing strains E. coli DH5 $\alpha \lambda$ pir and E. coli DH5 $\alpha \lambda$ pir containing pKK30.

Krute, C. N., Krausz, K. L., Markiewicz, M. A., Joyner, J. A., Pokhrel, S., Hall, P. R., et al. (2016). Generation of a stable plasmid for in vitro and in vivo studies of Staphylococcus species. Appl. Environ. Microbiol. 82, 6859-6869. doi: 10.1128/aem.02370-16

Silhavy, T. J., Berman, M. L., and Enquist, L. W. (1984). Experiments with Gene Fusions. Cold Spring Harbor, NY: Cold Spring Harbor Laboratory.

Zarate, M. A., Rodriguez, M. D., Chang, E. I., Russell, J. T., Arndt, T. J., Richards, E. M., et al. (2017). Post-hypoxia invasion of the fetal brain by multidrug resistant Staphylococcus. Sci. Rep. 7:6458. doi: 10.1038/s41598-01706789-6

Conflict of Interest Statement: The authors declare that the research was conducted in the absence of any commercial or financial relationships that could be construed as a potential conflict of interest.

Copyright (C) 2017 Rodriguez, Paul, Wood, Rice and Triplett. This is an open-access article distributed under the terms of the Creative Commons Attribution License (CC BY). The use, distribution or reproduction in other forums is permitted, provided the original author(s) or licensor are credited and that the original publication in this journal is cited, in accordance with accepted academic practice. No use, distribution or reproduction is permitted which does not comply with these terms. 